\title{
Flexible band versus rigid ring annuloplasty for tricuspid regurgitation: a systematic review and meta-analysis
}

\author{
Nelson Wang ${ }^{1}$, Steven Phan $^{1}$, David H. Tian ${ }^{2,3}$, Tristan D. Yan ${ }^{2}$, Kevin Phan ${ }^{1,2}$ \\ ${ }^{1}$ Sydney Medical School, University of Sydney, Sydney, Australia; ${ }^{2}$ Collaborative Research (CORE) Group, Macquarie University, Sydney, Australia; \\ ${ }^{3}$ Royal North Shore Hospital, Sydney, Australia \\ Correspondence to: Nelson Wang. Sydney Medical School, University of Sydney, Sydney, Australia. Email: nwan5905@uni.sydney.edu.au.
}

\begin{abstract}
Background: Up to $20 \%$ of patients have pre-discharge residual moderate to severe tricuspid regurgitation (TR) after tricuspid repair. Reoperations for recurrent TR carry high mortality rates, which emphasizes the importance of identifying the optimal technique for the surgical management of TR. The present study is a systematic review and meta-analysis that aims to compare short and long term survival and freedom from TR of flexible band ring versus rigid ring for annuloplasty of TR.

Methods: We conducted a systematic review and meta-analysis of comparative studies to evaluate these procedures. A systematic search of the literature was performed from six electronic databases. Pooled metaanalysis was conducted using odds ratio (OR) and weighted mean difference (WMD).

Results: The rates of in-hospital mortality were not different between the two groups, with cumulative rates of $6.9 \%$ for flexible band and $7.3 \%$ for rigid ring (OR: $0.92 ; 95 \%$ CI: $0.49-1.71$ ). Rates of stroke were also similar with $1.7 \%$ of flexible band and $1.3 \%$ of rigid rings suffering a perioperative stroke (OR: 1.29; $95 \%$ CI: 0.74-2.23). Rigid ring had significantly better freedom from grade $\geq 2$ TR at 5 years (OR: $0.44 ; 95 \%$ CI: $0.20-0.99)$ and overall $(\mathrm{P}=0.005)$. There was no significant difference in overall rates of reoperation $(\mathrm{P}=0.232)$ and survival $(\mathrm{P}=0.086)$ between flexible band and rigid ring.

Conclusions: Both rigid ring and flexible band offer acceptable outcomes for the treatment of TR. Compared to flexible band, rates of TR are stable after rigid ring annuloplasty and long term freedom from TR are superior for rigid ring devices. Large prospective randomized trials are required in order to validate these findings and assess for improvements in patient survival.
\end{abstract}

Keywords: Flexible band; rigid ring; tricuspid regurgitation

Submitted Mar 08, 2017. Accepted for publication Apr 18, 2017.

doi: $10.21037 /$ acs.2017.05.05

View this article at: http://dx.doi.org/10.21037/acs.2017.05.05

\section{Introduction}

Tricuspid regurgitation (TR) involves enlargement of the valve annulus and tethering of the leaflets, as a consequence of dilatation and/or dysfunction of the right sided heart chambers $(1,2)$. Although mild TR is usually insignificant, moderate-severe TR is an independent risk factor for progressive heart failure and increased mortality (3-6). Tricuspid valve annuloplasty was traditionally treated with the classic De Vega annuloplasty but has since evolved after the development of prosthetic tricuspid annuloplasty.

Up to $20 \%$ of patients have pre-discharge residual moderate to severe TR after tricuspid repair(7-9).
Reoperations for recurrent TR carry high mortality rates (10), which emphasises the importance of identifying the optimal technique for the surgical management of TR. Head-to-head comparisons have demonstrated superior long term outcomes with device-based annuloplasty compared to suture-based surgery $(11,12)$. There are three primary devices employed; standard rigid rings that were predominant in the 1990s, flexible bands which became increasingly employed from the early 2000s and threedimensional rigid rings in recent years (13).

Current evidence in the comparison of these devices has been limited to cohort studies with no clear consensus on a 
superior device $(8,9,14)$. The present study is a systematic review and meta-analysis that aims to compare the outcomes of flexible band ring versus rigid ring for annuloplasty of TR.

\section{Methods}

\section{Search strategy}

The PRISMA statement and recommended guidelines were followed for the present systematic review and metaanalysis $(15,16)$. Electronic searches were performed using Ovid Medline, PubMed, Cochrane Central Register of Controlled Trials (CCTR), Cochrane Database of Systematic Reviews (CDSR), ACP Journal Club and Database of Abstracts of Review of Effectiveness (DARE) from their dates of inception to December 2016. To achieve maximum sensitivity of the search strategy and identify all studies, we combined the terms: "tricuspid valve", "tricuspid regurgitation", "annuloplasty", "rigid-ring" or "flexiblering" as either keywords or MeSH terms. The reference lists of all retrieved articles were reviewed for further identification of potentially relevant studies. All identified articles were systematically assessed using the inclusion and exclusion criteria.

\section{Selection criteria}

Eligible comparative studies for the present systematic review and meta-analysis included those in which patient cohorts underwent rigid-ring versus flexible-ring annuloplasty for tricuspid valve regurgitation. Studies that did not include SR or AF-free survival as endpoints were excluded. When institutions published duplicate studies with accumulating numbers of patients or increased lengths of follow-up, only the most complete reports were included for quantitative assessment at each time interval. All publications were limited to those involving human subjects and in the English language. Abstracts, case reports, conference presentations, editorials and expert opinions were excluded. Review articles were omitted because of potential publication bias and duplication of results.

\section{Data extraction and critical appraisal}

All data were extracted from article texts, tables and figures. Two investigators independently reviewed each retrieved article (N.W., K.P.). Discrepancies between the two reviewers were resolved by discussion and consensus. Assessment of risk of bias for each selected study was performed according to the most updated Cochrane statement.

\section{Statistical analysis}

The odds ratio (OR) was used as a summary statistic. In the present study, both fixed- and random-effect models were tested. In the fixed-effects model, it was assumed that treatment effect in each study was the same, whereas in a random-effects model, it was assumed that there were variations between studies. $\chi^{2}$ tests were used to study heterogeneity between trials. $\mathrm{I}^{2}$ statistic was used to estimate the percentage of total variation across studies, owing to heterogeneity rather than chance, with values greater than $50 \%$ considered as substantial heterogeneity. $\mathrm{I}^{2}$ can be calculated as: $\mathrm{I}^{2}=100 \% \times(\mathrm{Q}-\mathrm{df}) / \mathrm{Q}$, with $\mathrm{Q}$ defined as Cochrane's heterogeneity statistics and $\mathrm{df}$ defined as degree of freedom. If there was substantial heterogeneity, the possible clinical and methodological reasons for this were explored qualitatively. In the present meta-analysis, the results using the random-effects model were presented to take into account the possible clinical diversity and methodological variation between studies. Specific analyses considering confounding factors were not possible because raw data were not available. All $\mathrm{P}$ values were two-sided. Pooled meta-analysis was conducted with Review Manager Version 5.3.2 (Cochrane Collaboration, Software Update, Oxford, United Kingdom).

For long-term outcomes presented by Kaplan-Meier analysis in the included studies, individual patient survival data was reconstructed using an iterative algorithm that was applied to solve the Kaplan-Meier equations originally used to produce the published graphs. This algorithm, as provided by Guyot and colleagues, uses digitalized KaplanMeier curve data to find numerical solutions to the inverted Kaplan-Meier equations (17). This algorithm assumes constant censoring and was calculated in $\mathrm{R}$ software (v.3.1.0). The reconstructed patient survival data were then aggregated to form combined survival curves.

\section{Publication bias}

Evidence of publication bias was sought using the methods of funnel plot symmetry. If studies appear to be missing in areas of low statistical significance, then it is possible that the asymmetry is due to publication bias. If studies appear to be missing in areas of high statistical significance, then publication bias is a less likely cause of funnel asymmetry. 


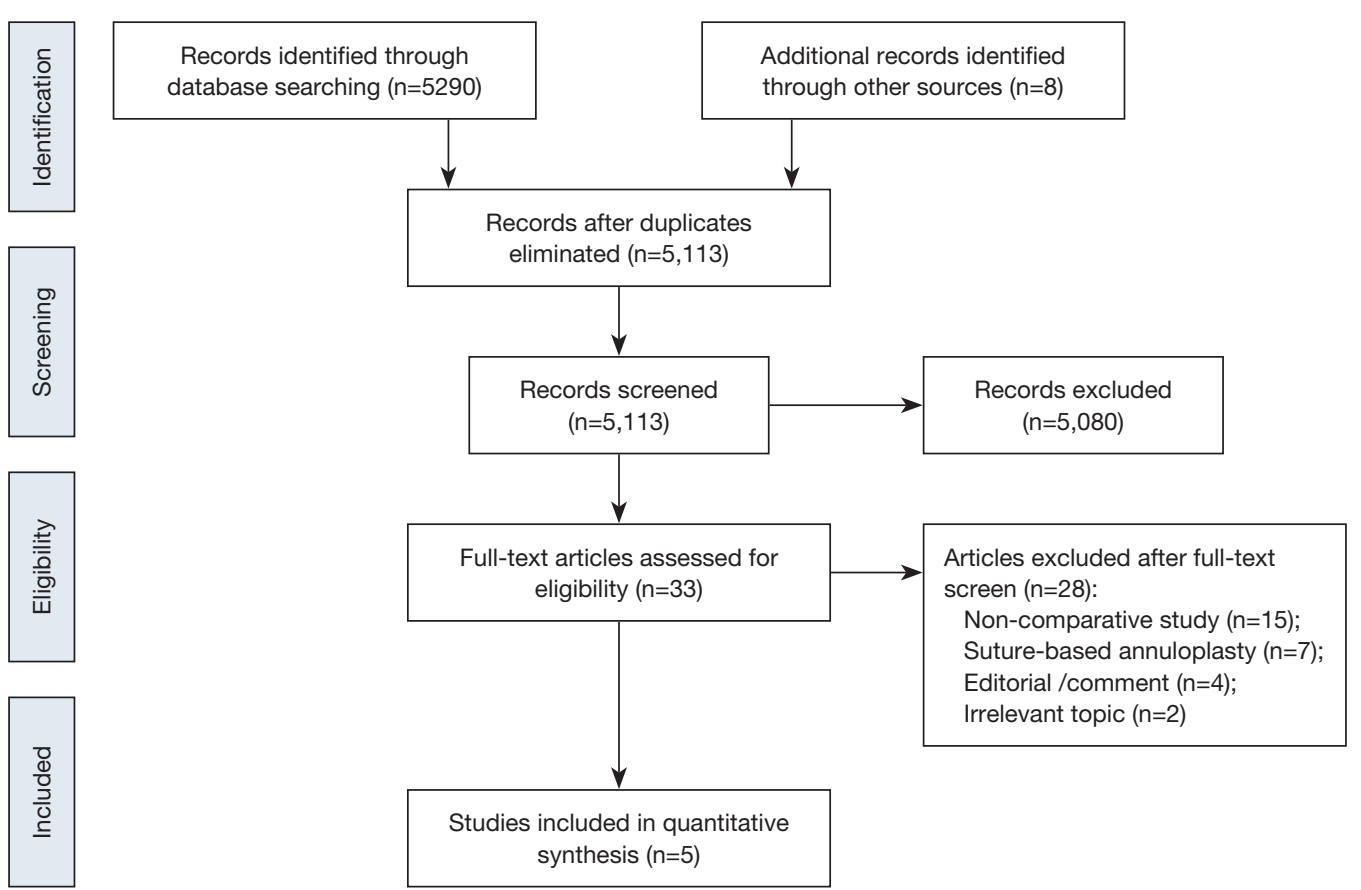

Figure 1 Study selection. Flow chart showing the results in each systematic search to identify studies comparing flexible band and rigid ring prosthesis.

\section{Results}

\section{Study characteristics}

Our search strategy identified 5,113 studies and after title and abstract screen, 33 were included for full text review (Figure 1). We identified six studies $(8,9,14,18-20)$ that met our inclusion criteria, of which one study was excluded from analysis due to overlapping population (9). Finally, we included a total of five studies with 3,141 patients of which $1,893 \mathrm{had}$ a flexible band and 1,248 had a rigid ring. All studies were observational and the mean duration of followup ranged from 1.8 to 5.7 years. One study exclusively evaluated patients undergoing tricuspid valve replacement with concomitant mitral or aortic valve surgery (8), whilst another study only included patients with TR and rheumatic mitral valve disease (20) (Table 1). There were differences in the inclusion criteria based on degree of TR. One study included patients with at least mild TR (18), two of the studies included patients with at least moderate TR $(19,20)$, one study included patients with severe TR or moderate TR requiring other cardiac surgery (14) and one study did not specify the degree of TR but all the patients had concomitant aortic or mitral valve surgery (8). Among the five studies, only three provided information for analysis of long-term follow-up $(8,14,18)$.

\section{Baseline characteristics}

Regardless of type of annuloplasty, the majority of the patients requiring tricuspid surgery were elderly, with four of the five studies having mean age greater than 68 years. One study had a younger cohort of mean age 57 years. Patients were predominantly female (48\% to $60 \%$ ) and a significant proportion had atrial fibrillation (26\% to $86 \%$ ). The majority of the patients had moderate to severe TR at baseline (Table 2). Although patients undergoing rigid rings were slightly older (Mean Difference: 1.15; $95 \%$ CI: 0.21-2.09), a greater proportion of patients using a flexible band had atrial fibrillation (OR: $1.30 ; 95 \%$ CI: $1.03-1.64)$ and a permanent pacemaker (OR: 1.72; $95 \%$ CI: 1.16-2.57). There was a trend for patients undergoing rigid ring annuloplasty to have a higher EuroSCORE II (mean difference: 1.52 ; $95 \%$ CI: $0.36-3.41$ ), but this was not significant. In one study, more patients undergoing rigid rings had peripheral vascular disease (OR: $0.51 ; 95 \%$ CI: 0.29-0.89) and heart failure (OR: 0.44; 95\% CI: 0.27-0.72). There was no significant difference in rates of moderate or severe TR at baseline (Figure S1). 

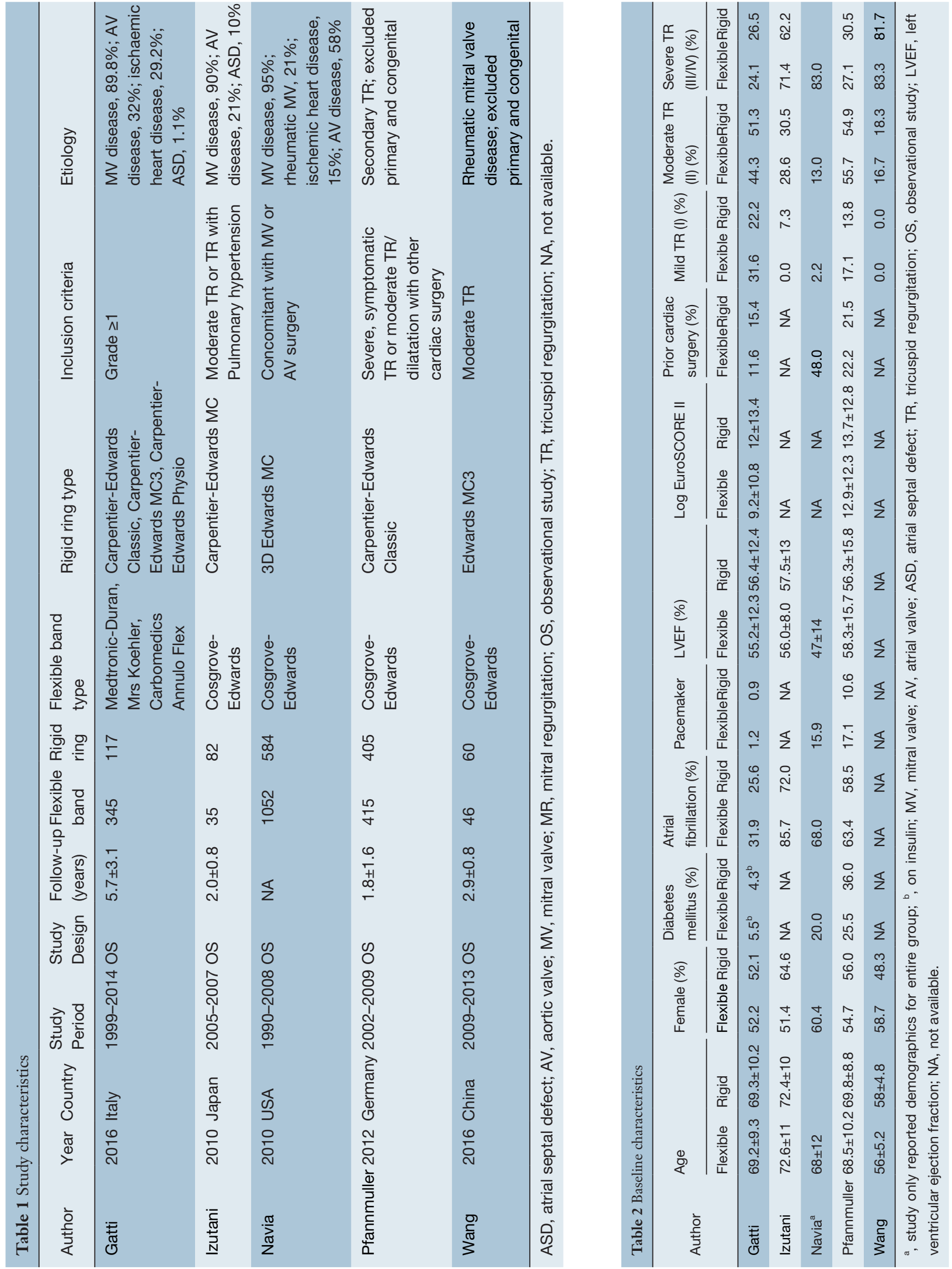


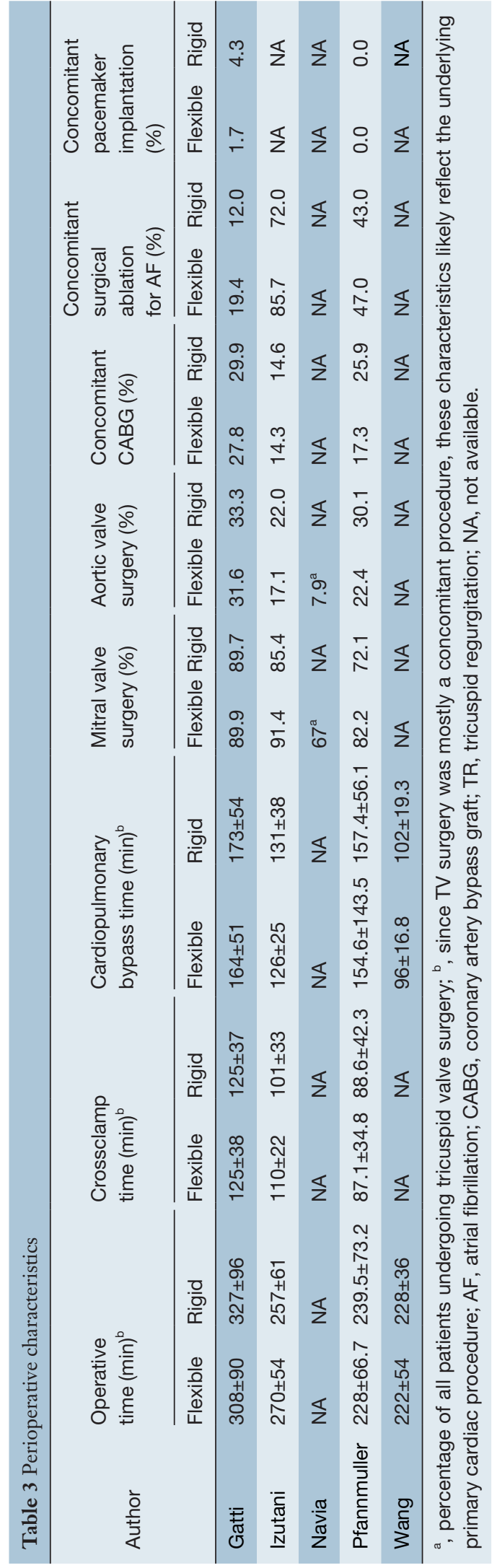

\section{Perioperative characteristics}

The mean operative time ranged from 222 to 308 minutes for flexible band and 228 to 327 minutes for rigid ring (Table 3). A significant proportion of both groups required concomitant mitral and/or aortic valve surgery. Compared to flexible band, rigid ring annuloplasty had a non-significant trend for longer operation times (mean difference: 7.62; 95\% CI: 3.14-18.38). Cardiopulmonary bypass time was significantly longer for rigid ring procedures (mean difference: 6.05; 95\% CI: 1.11-10.99). A large number of the TV procedures were concomitant with other cardiac surgeries and hence the above operative characteristics likely reflect the primary surgical procedure. The main underlying cardiac surgeries performed were mitral and aortic valve surgery, CABG and surgical ablation for atrial fibrillation. More flexible band procedures had concomitant mitral valve (OR: $1.50 ; 95 \%$ CI: $1.15-1.96$ ) but less aortic valve surgery (OR: 0.78; 95\% CI: 0.63-0.97) and CABG (OR: 0.72; 95\% CI: 0.53-0.97). Although not significant, there was a trend for flexible band surgery to undergo surgical ablation for atrial fibrillation (OR: 1.39; 95\% CI: 0.98-1.98) (Figure S2).

\section{Outcomes}

The rates of in-hospital mortality were not different between the two groups, with cumulative rates of $6.9 \%$ for flexible band and $7.3 \%$ for rigid ring (OR: 0.92; 95\% CI: 0.49-1.71). Rates of stroke were also similar with $1.7 \%$ of flexible band and $1.3 \%$ of rigid rings suffering a perioperative stroke (OR: 1.29; 95\% CI: 0.74-2.23) (Figure 2). Rigid ring had significantly better freedom from grade $\geq 2 \mathrm{TR}$ at 5 years (OR: 0.44; 95\% CI: 0.20-0.99) but not at 1 year (OR: 0.51; 95\% CI: $0.20-1.28$ ) and 3 years (OR: 0.45 ; 95\% CI: $0.17-1.16$ ) (Figure 3). Overall freedom from grade $\geq 2 \mathrm{TR}$ was better in the rigid ring group $(\mathrm{P}=0.005)$ (Figure 4). Freedom from reoperation and survival were similar at 1,3 and 5 years (Figure S3). There was no significant difference in overall rates of reoperation $(\mathrm{P}=0.232)$ and survival $(\mathrm{P}=0.086)$ between flexible band and rigid ring (Figure 4).

\section{Publication bias}

Funnel plots for 5-year freedom from reoperation, survival and freedom from recurrent TR was assessed (Figure S4). No significant funnel plot asymmetry was noted, suggesting publication bias was not a significant influencing factor. 


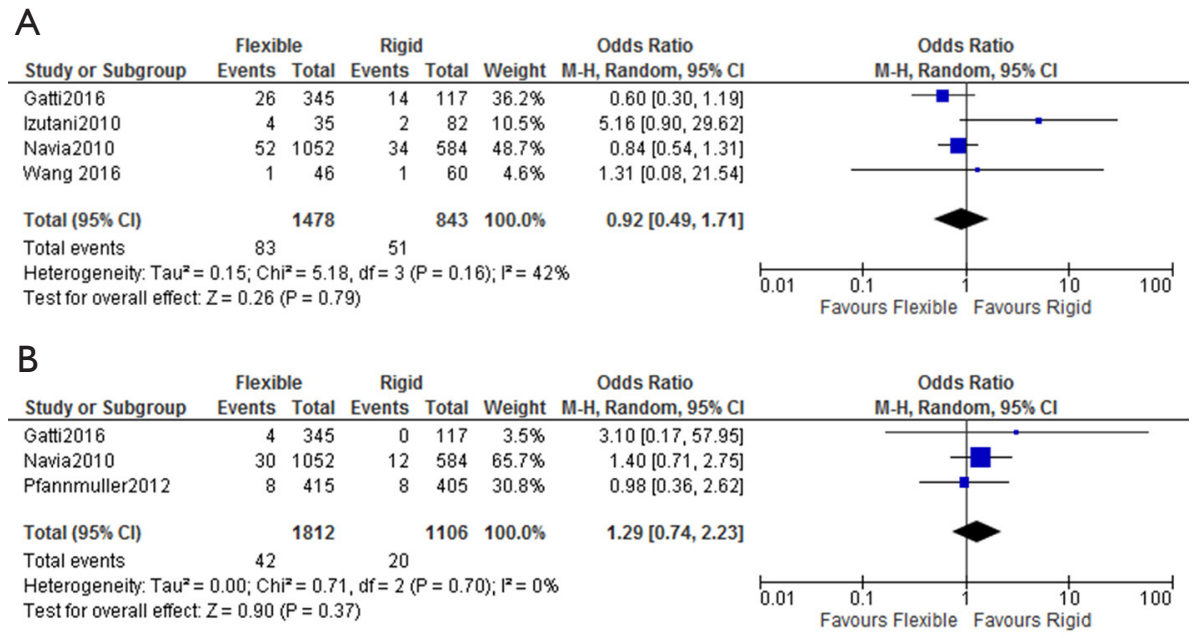

Figure 2 Perioperative outcomes after flexible band and rigid ring annuloplasty. Forest plot of crude odds ratio for (A) in hospital mortality and (B) strokes in patients undergoing tricuspid valve surgery with flexible band or rigid ring prosthesis.

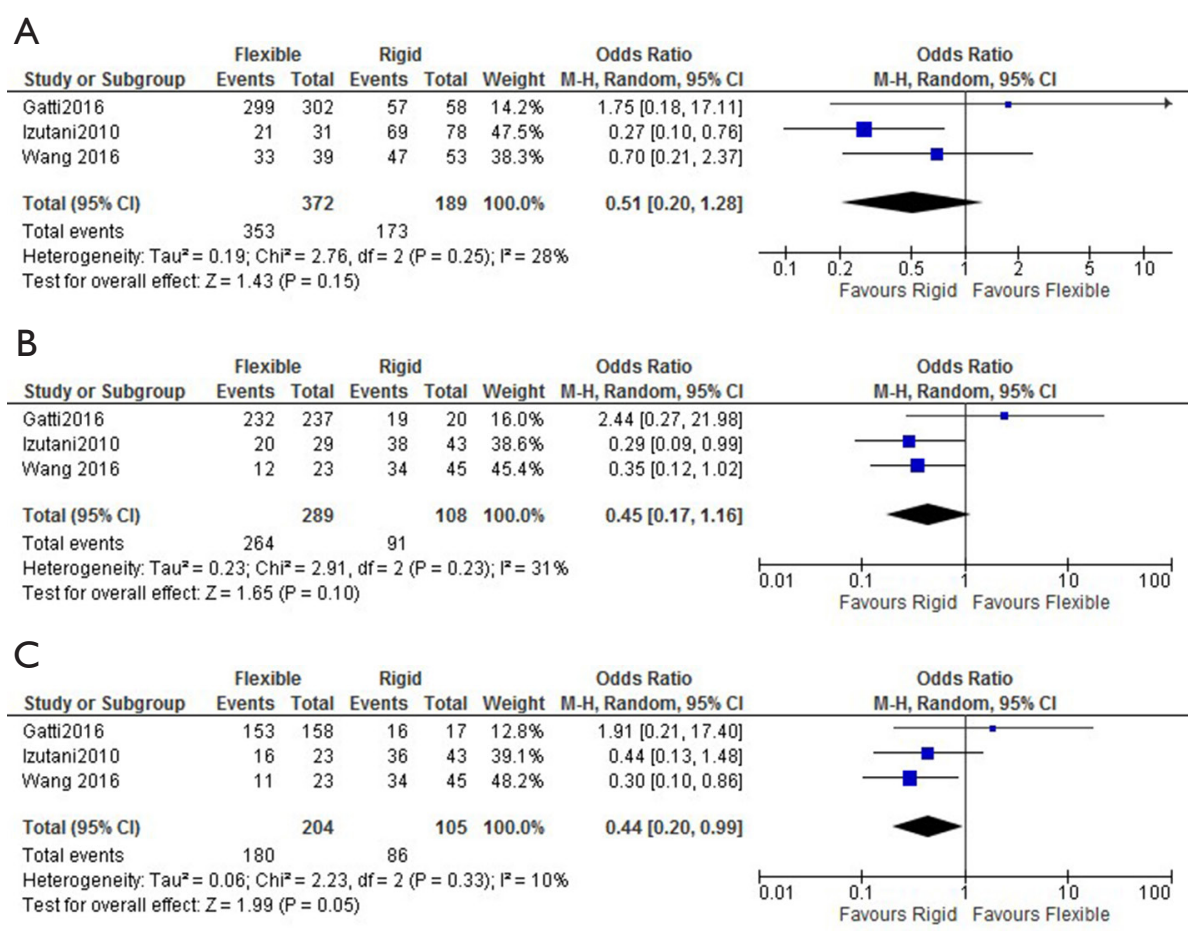

Figure 3 Freedom from tricuspid regurgitation grade $\geq 2$ in patients undergoing flexible band and rigid ring annuloplasty. Comparison of rates of freedom from tricuspid regurgitation grade $\geq 2$ at (A) 1 year, (B) 3 years and (C) 5 years post operation.

\section{Discussion}

We have shown that both flexible band and rigid ring annuloplasty generate acceptable short term and long term results for the surgical management of TR. The grade of
TR was relatively stable post operation in patient with a rigid ring prosthesis. In contrast, there was slow but gradual increase in rates of TR with the flexible band. The freedom from grade $\geq 2$ TR was similar at 1 and 3 years, however 

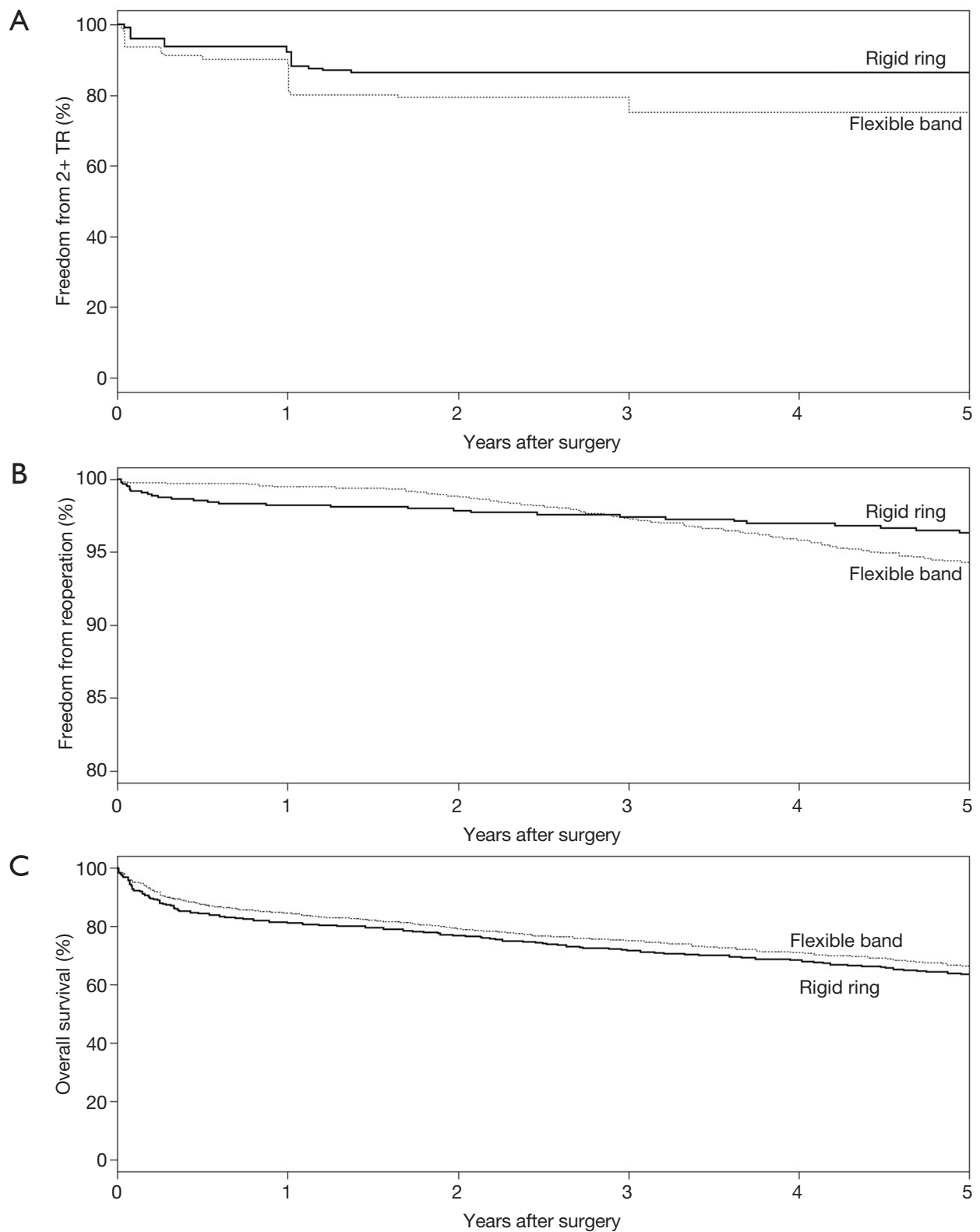

Figure 4 Long term outcomes after flexible band and rigid ring. Kaplan-Meier curves comparing long term (A) freedom from tricuspid regurgitation grade $\geq 2$, (B) freedom from reoperation and (C) survival in patients undergoing flexible band and rigid ring annuloplasty. Rigid ring had significantly greater freedom from grade $\geq 2$ tricuspid regurgitation $(\mathrm{P}=0.005)$. There was no significant difference in freedom from reoperation $(\mathrm{P}=0.232)$ and survival $(\mathrm{P}=0.086)$. TR, tricuspid regurgitation.

rigid ring had significantly better freedom from TR at 5 years and overall. Despite this, there was no difference in reoperation rates and survival between the two groups.

Moderate-severe degrees of TR are best treated with bands or rings $(2,6,7,21)$, with suture annuloplasty primarily reserved for mild-moderate cases $(11,12)$. Three types of devices have been devised and implemented throughout the years, including standard rigid rings, flexible bands and $3 \mathrm{D}$ rigid rings (8). The innovation of the bands in the early 2000s were driven by their inherent advantage of improved 
flexibility. The band's flexibility offers a simpler design and implantation technique, carrying lower risks of device breakages and tricuspid stenosis after device implantation. The bands also carry negligible risk of injuring the cardiac tissue and nearby coronary vessels during the operation. Flexible bands can also best preserve RV function and assist RV functional recovery after surgery (22-24). Pfannmuller et al. concluded that flexible bands had significantly less ring dehiscence than patients with a rigid ring (14). This may be due to greater forces present on the sutures attached to a rigid ring compared to the flexible band that might more effectively follow the natural motion of the tricuspid annulus. Fracture of the ring has also been reported by other studies as an infrequent complication of the rigid ring in the tricuspid position $(25,26)$.

Despite the potential advantages of flexible band, there have been no reports of superior outcomes with bands versus rings. In fact there have been reports that rings can have lower rates of recurrent TR than flexible bands, although both adequately control TR $(9,24)$. Previous studies have reported that the grade of TR after rigid ring is relatively stable across time whilst slowly increasing after flexible band $(8,9,20)$. Our results are consistent with these findings, as significant differences in freedom from TR were only seen after long term follow-up.

The normal tricuspid annulus is saddle-shaped, with the highest points in anteroposterior orientation and lowest points in mediolateral orientation. The most common cause of TR is left sided valve disease, which causes the annulus to become dilated, flattened, and circular $(27,28)$. Fukuda et al. has demonstrated an asymmetric reduction in tricuspid annular contraction in patients with secondary TR, which suggests annular dilatation predisposes a patient to future TR (7). Secondary remodeling of the RV may also be a factor for TR recurrence post operation (8).

The flexible bands are designed to allow natural physiological motion of the tricuspid annulus throughout the cardiac cycle but this may hinder optimal positioning and maintenance of the saddle annulus (20). The flexible band is implanted from the anteroseptal to posteroseptal commissure, whereas the rigid ring is implanted from the anteroseptal commissure to the middle of the septal leaflet. By not implanting the band up to the middle of the septal leaflet, the attachments of the band may be compromised which leads to increased rates of TR. In contrast, the three-dimensional model of the rigid ring is designed to accommodate the saddle shaped annulus. Due to the novelty and complexity of the procedure, surgical techniques for flexible bands have not been as standardized compared to rigid ring. Variations in implantation technique of the flexible bands may contribute to some of the increased TR rates.

There were some differences in the baseline features of the two groups. Patients undergoing rigid ring annuloplasty were slightly older and had a trend for a higher EuroSCORE II. Patients undergoing flexible bands had greater rates of atrial fibrillation and more patients had a permanent pacemaker. The larger number of pacemakers in the flexible band group could also partly contribute to the elevated TR rates over time. Mitral valve stenosis or regurgitation is the most common cause of TR as it causes annular dilation and leaflet tethering. Therefore, correction of the left sided valve disease is often essential to limit the back pressures on the right ventricle. Concomitant mitral surgery was common in both flexible band and rigid ring (29). Concomitant mitral valve surgery was required more frequently in the flexible band, which may be reflective of the increased rates of atrial dilatation and atrial fibrillation. More aortic valve surgery was performed with rigid ring, which may be associated with an older patient group.

\section{Limitations}

This study has several limitations to be considered. The analysis of long term outcomes has been limited to three studies and our results may not have been powered to detect differences in reoperation and survival. All studies were observational in design and are subject to selection bias that may have influenced the results. Different inclusion criteria, operative techniques and devices may also contribute to heterogeneity between studies. Future randomized studies with long term follow-up are required in order to validate our findings.

\section{Conclusions}

Both rigid ring and flexible band offer acceptable outcomes for the treatment of TR. Compared to flexible band, rates of TR are stable after rigid ring annuloplasty and long term freedom from TR may be superior for rigid ring devices. Large prospective randomized trials are required in order to validate these findings and assess for improvements in patient survival.

\section{Acknowledgements}

None. 


\section{Footnote}

Conflicts of Interest: The authors have no conflicts of interest to declare.

\section{References}

1. Goldstone AB, Howard JL, Cohen JE, et al. Natural history of coexistent tricuspid regurgitation in patients with degenerative mitral valve disease: implications for future guidelines. J Thorac Cardiovasc Surg 2014;148:2802-9.

2. Di Mauro M, Bezante GP, Di Baldassarre A, et al. Functional tricuspid regurgitation: an underestimated issue. Int J Cardiol 2013;168:707-15.

3. Koelling TM, Aaronson KD, Cody RJ, et al. Prognostic significance of mitral regurgitation and tricuspid regurgitation in patients with left ventricular systolic dysfunction. Am Heart J 2002;144:524-9.

4. Nath J, Foster E, Heidenreich PA. Impact of tricuspid regurgitation on long-term survival. J Am Coll Cardiol 2004;43:405-9.

5. Antunes MJ, Barlow JB. Management of tricuspid valve regurgitation. Heart 2007;93:271-6.

6. Filsoufi F, Anyanwu AC, Salzberg SP, et al. Long-term outcomes of tricuspid valve replacement in the current era. Ann Thorac Surg 2005;80:845-50.

7. Fukuda S, Gillinov AM, McCarthy PM, et al. Echocardiographic follow-up of tricuspid annuloplasty with a new three-dimensional ring in patients with functional tricuspid regurgitation. J Am Soc Echocardiogr 2007;20:1236-42.

8. Navia JL, Nowicki ER, Blackstone EH, et al. Surgical management of secondary tricuspid valve regurgitation: annulus, commissure, or leaflet procedure? J Thorac Cardiovasc Surg 2010;139:1473-82.e5.

9. McCarthy PM, Bhudia SK, Rajeswaran J, et al. Tricuspid valve repair: durability and risk factors for failure. J Thorac Cardiovasc Surg 2004;127:674-85.

10. Jeganathan R, Armstrong S, Al-Alao B, et al. The risk and outcomes of reoperative tricuspid valve surgery. Ann Thorac Surg 2013;95:119-24.

11. Huang X, Gu C, Men X, et al. Repair of functional tricuspid regurgitation: comparison between suture annuloplasty and rings annuloplasty. Ann Thorac Surg 2014;97:1286-92.

12. Parolari A, Barili F, Pilozzi A, et al. Ring or suture annuloplasty for tricuspid regurgitation? A meta-analysis review. Ann Thorac Surg 2014;98:2255-63.
13. Starck CT, Kempfert J, Falk V. Tricuspid valve interventions: surgical techniques and outcomes. EuroIntervention 2015;11 Suppl W:W128-32.

14. Pfannmuller B, Doenst T, Eberhardt K, et al. Increased risk of dehiscence after tricuspid valve repair with rigid annuloplasty rings. J Thorac Cardiovasc Surg 2012;143:1050-5.

15. Moher D, Liberati A, Tetzlaff J, et al. Preferred reporting items for systematic reviews and meta-analyses: the PRISMA statement. PLoS Med 2009;6:e1000097.

16. Phan K, Tian DH, Cao C, et al. Systematic review and meta-analysis: techniques and a guide for the academic surgeon. Annals of Cardiothoracic Surgery 2015;4:112-22.

17. Guyot P, Ades AE, Ouwens MJ, et al. Enhanced secondary analysis of survival data: reconstructing the data from published Kaplan-Meier survival curves. BMC Med Res Methodol 2012;12:9.

18. Gatti G, Dell'Angela L, Morosin M, et al. Flexible band versus rigid ring annuloplasty for functional tricuspid regurgitation: two different patterns of right heart reverse remodelling. Interact Cardiovasc Thorac Surg 2016;23:79-89.

19. Izutani H, Nakamura T, Kawachi K. Flexible band versus rigid ring annuloplasty for functional tricuspid regurgitation. Heart Int 2010;5:e13.

20. Wang H, Liu X, Wang X, et al. Comparison of outcomes of tricuspid annuloplasty with 3D-rigid versus flexible prosthetic ring for functional tricuspid regurgitation secondary to rheumatic mitral valve disease. J Thorac Dis 2016;8:3087-95.

21. Shiran A, Sagie A. Tricuspid regurgitation in mitral valve disease incidence, prognostic implications, mechanism, and management. J Am Coll Cardiol 2009;53:401-8.

22. Gatti G, Marciano F, Antonini-Canterin F, et al. Tricuspid valve annuloplasty with a flexible prosthetic band. Interact Cardiovasc Thorac Surg 2007;6:731-5.

23. Jung SH, Je HG, Song JM, et al. Outcomes following use of a modified Duran ring tricuspid valve reconstruction procedure for secondary tricuspid regurgitation. Circ J 2010;74:925-30.

24. Zhu TY, Wang JG, Meng X. Is a rigid tricuspid annuloplasty ring superior to a flexible band when correcting secondary tricuspid regurgitation? Interact Cardiovasc Thorac Surg 2013;17:1009-14.

25. Galinanes M, Duarte J, de Caleya DF, et al. Fracture of the Carpentier-Edwards ring in tricuspid position: a report of three cases. Ann Thorac Surg 1986;42:74-6.

26. Kay HR, Hammond GL. Fracture of a prosthetic tricuspid 
annular ring. J Thorac Cardiovasc Surg 1982;83:635.

27. Sukmawan R, Watanabe N, Ogasawara Y, et al. Geometric changes of tricuspid valve tenting in tricuspid regurgitation secondary to pulmonary hypertension quantified by novel system with transthoracic real-time 3-dimensional echocardiography. J Am Soc Echocardiogr 2007;20:470-6. 28. Ton-Nu TT, Levine RA, Handschumacher MD,

Cite this article as: Wang N, Phan S, Tian DH, Yan TD, Phan K. Flexible band versus rigid ring annuloplasty for tricuspid regurgitation: a systematic review and meta-analysis. Ann Cardiothorac Surg 2017;6(3):194-203. doi: 10.21037/ acs.2017.05.05 et al. Geometric determinants of functional tricuspid regurgitation: insights from 3-dimensional echocardiography. Circulation 2006;114:143-9.

29. Shinn SH, Dayan V, Schaff HV, et al. Outcomes of ring versus suture annuloplasty for tricuspid valve repair in patients undergoing mitral valve surgery. $\mathrm{J}$ Thorac Cardiovasc Surg 2016;152:406-15.e3. 


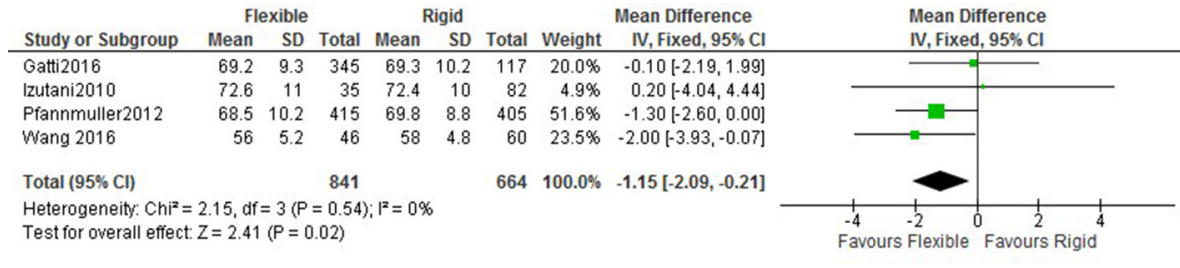

$\mathrm{D}$

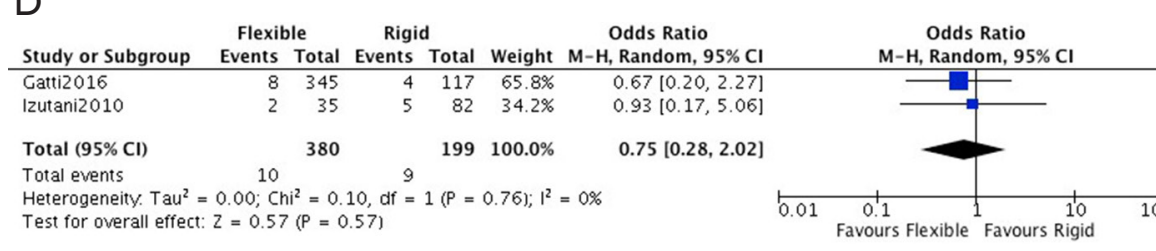

G

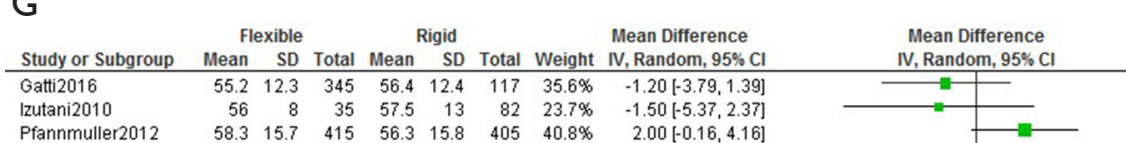

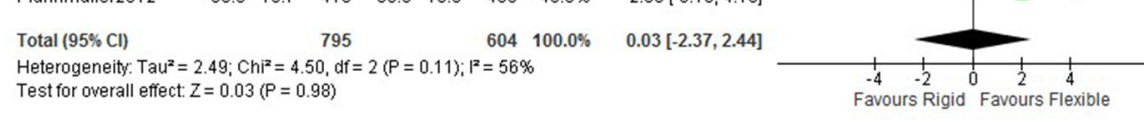

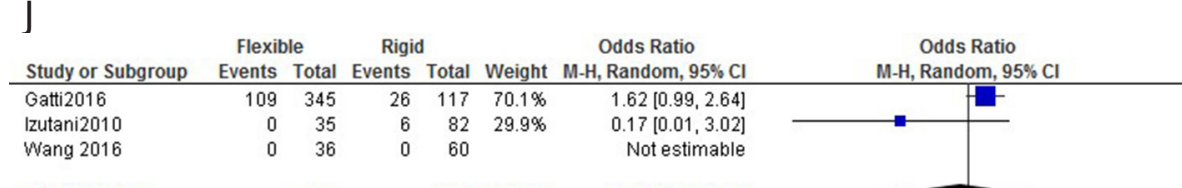

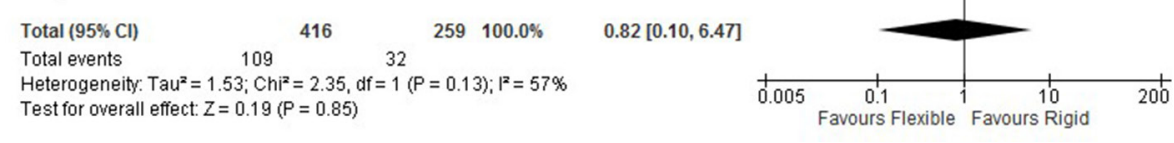

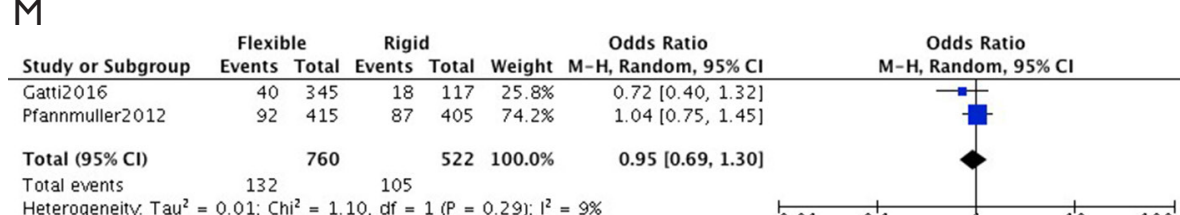

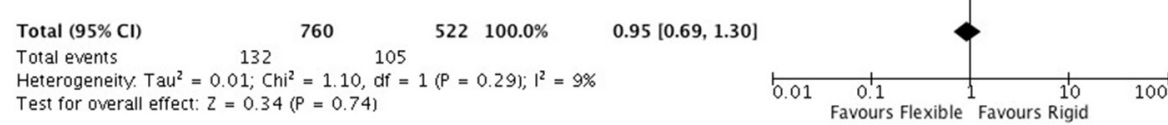

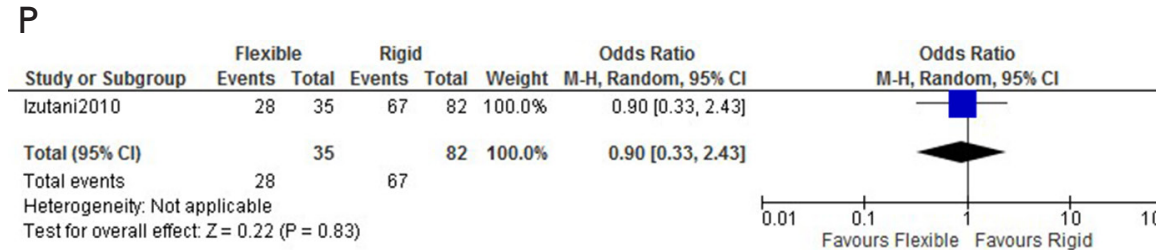

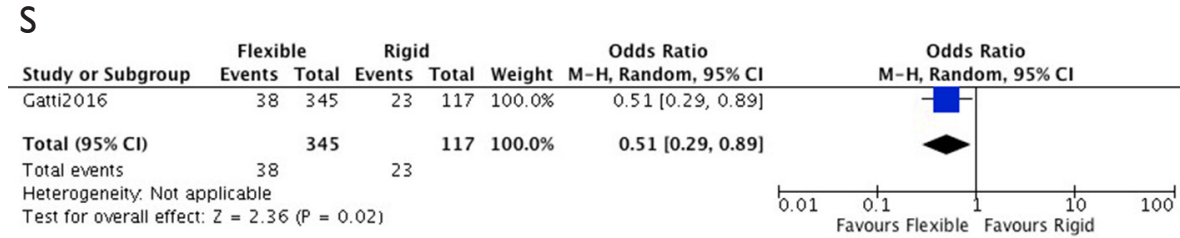

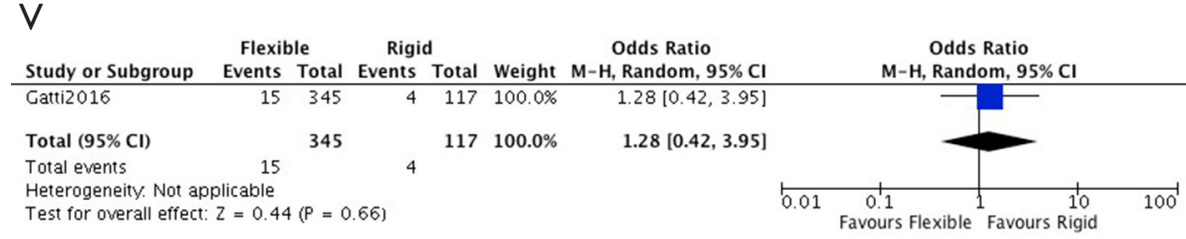

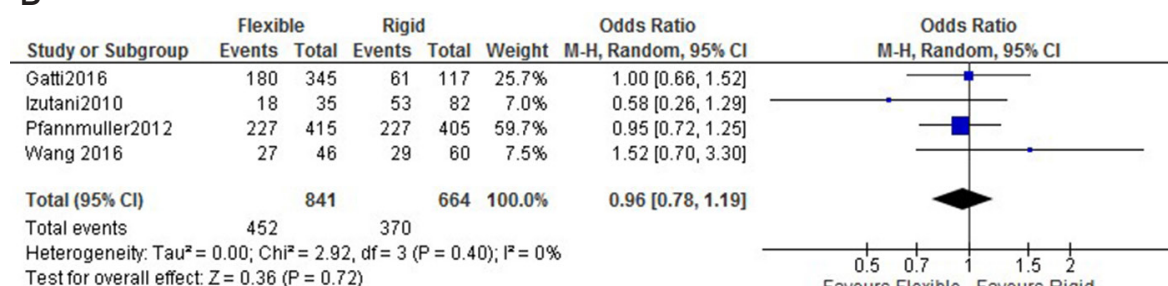
Favours fiexble Favours Rigid

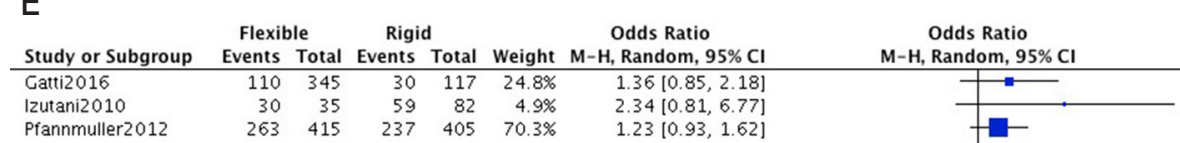

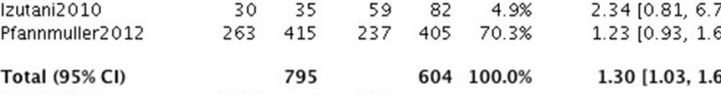

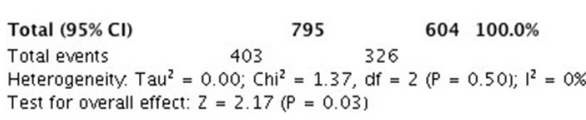

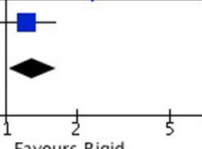

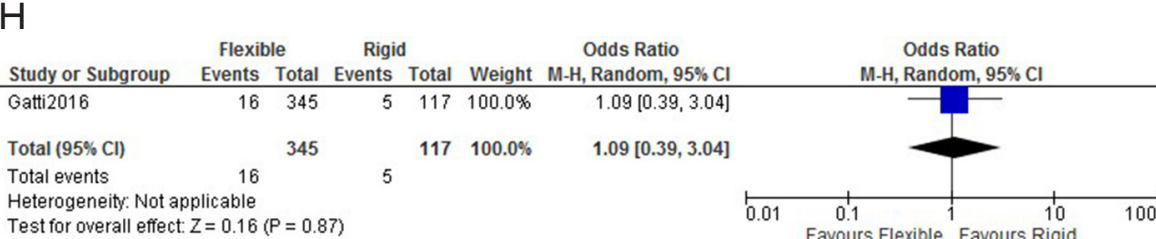

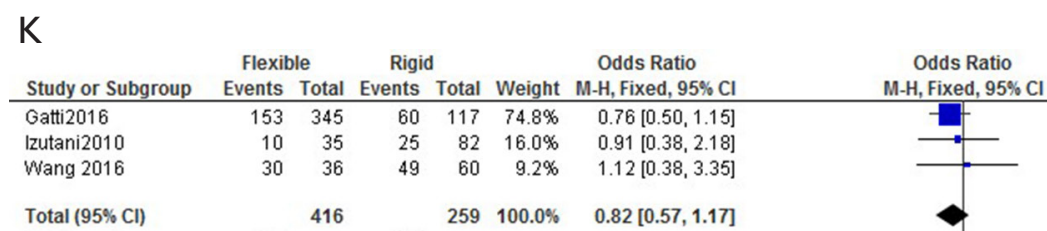

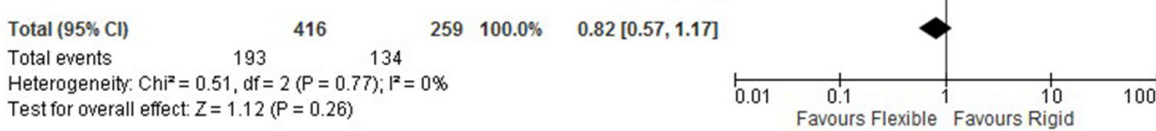

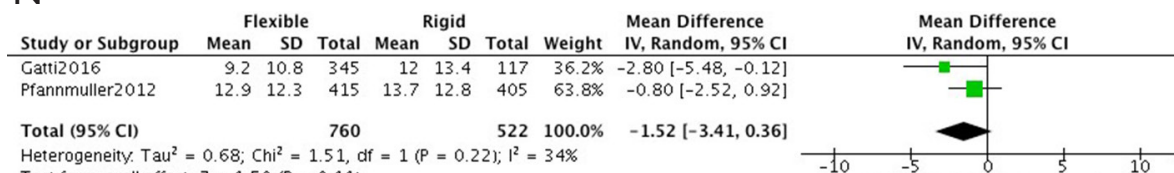

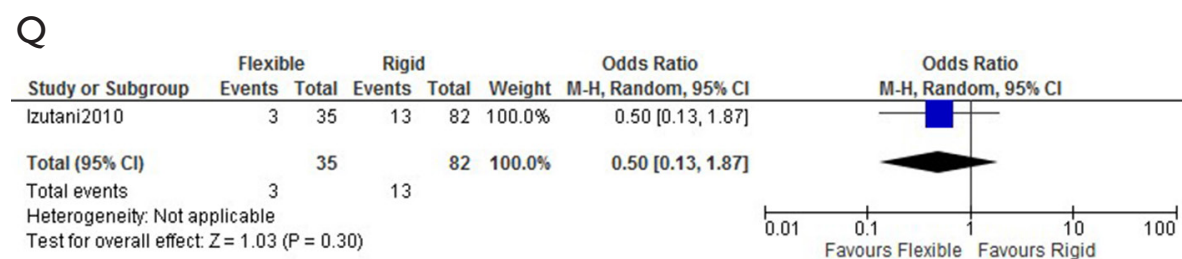

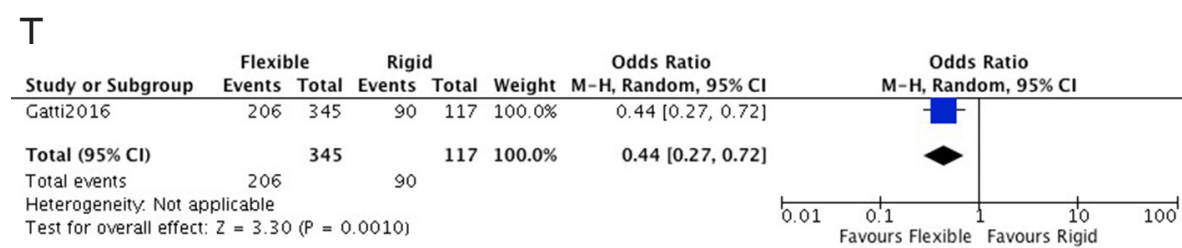

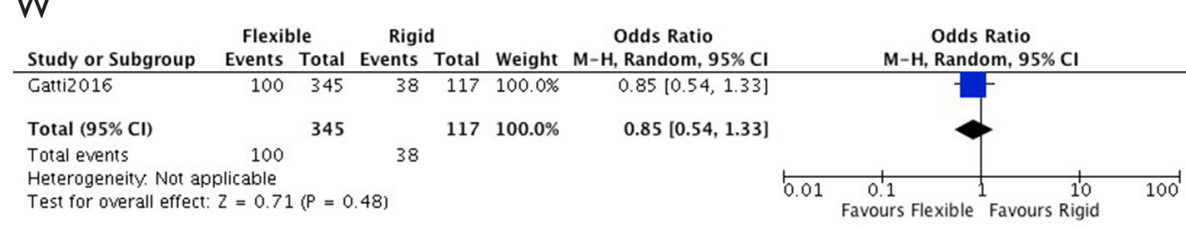

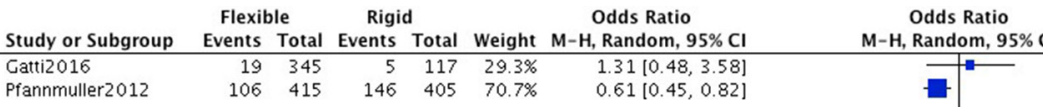

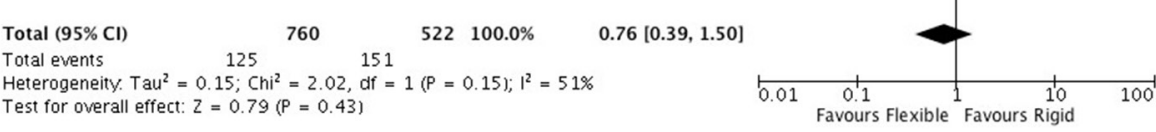

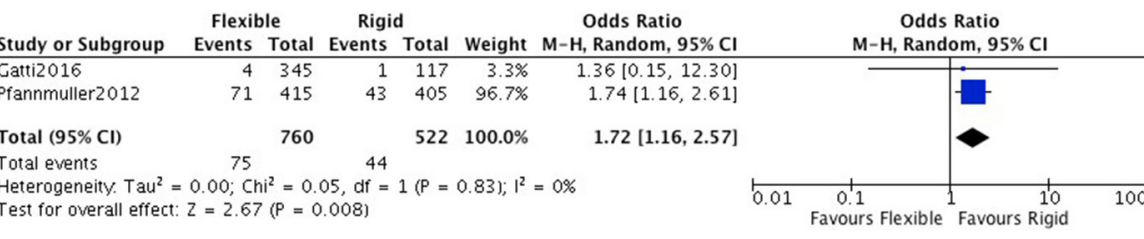

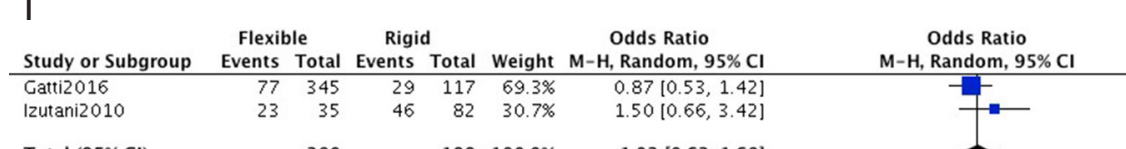

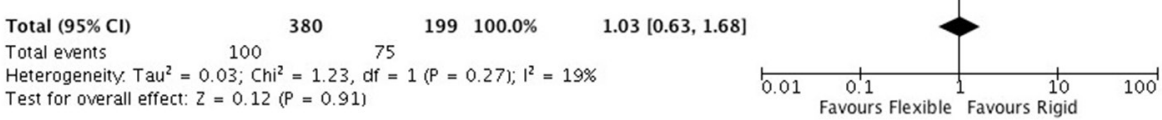

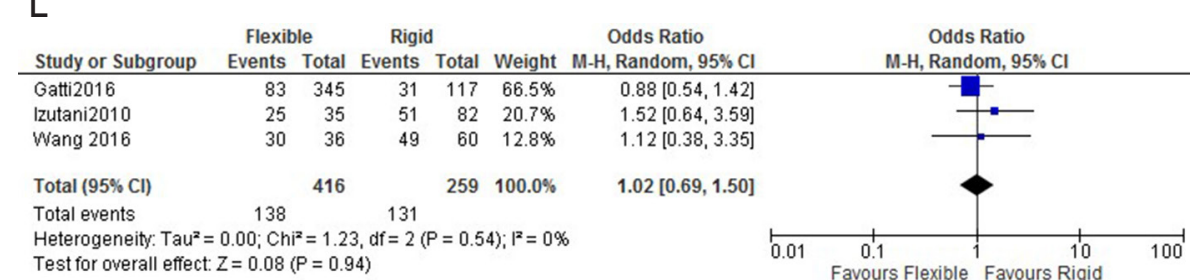

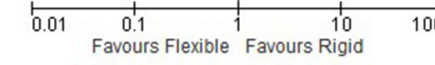

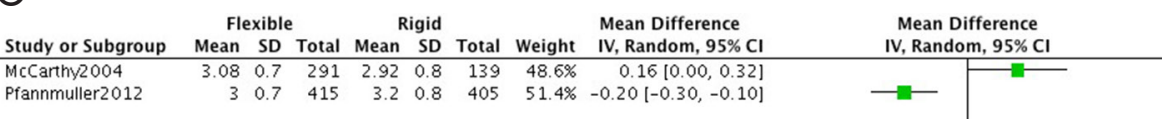

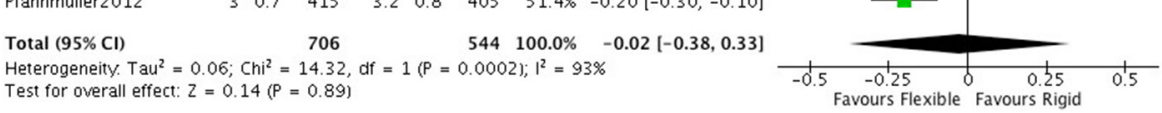

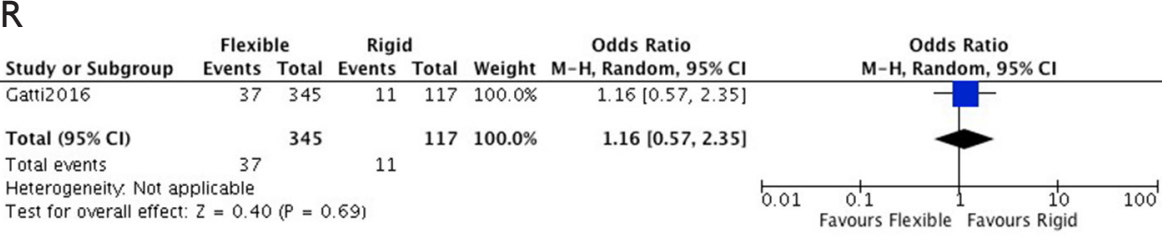

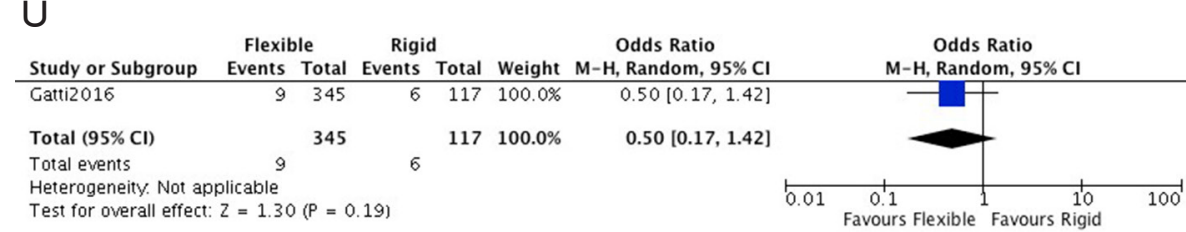

Figure S1 Comparison of baseline characteristics in flexible band and rigid ring. (A) Age; (B) females; (C) diabetes; (D) dialysis use; (E) atrial fibrillation; (F) permanent pacemaker; (G) left ventricular rejection fraction (\%); (H) LVEF <30\%; (I) pulmonary hypertension; (J) mild tricuspid regurgitation; (K) moderate tricuspid regurgitation; (L) severe tricuspid regurgitation; (M) prior cardiac surgery; (N) EuroSCORE; II; (O) NYHA class; (P) mitral regurgitation; (Q) aortic regurgitation; (R) chronic lung disease; (S) peripheral vascular disease; (T) heart failure; (U) peripheral edema; (V) recent myocardial moderate tricuspid regurgitation; (L) sever
infarction; $(\mathrm{W})$ coronary artery disease.

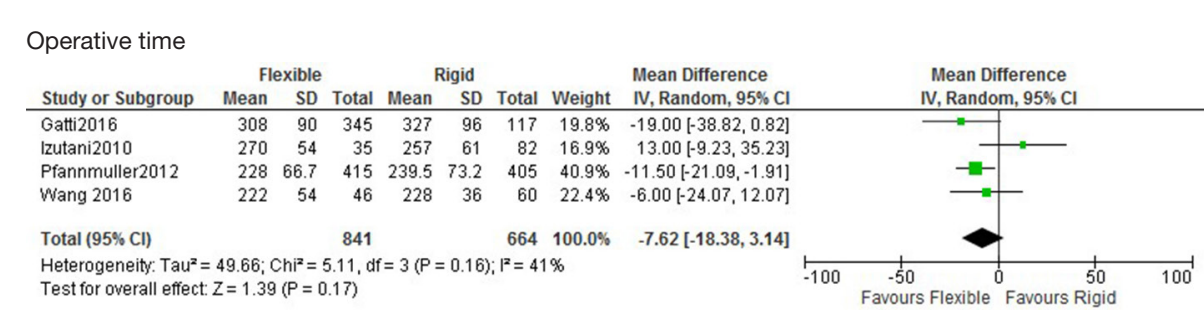

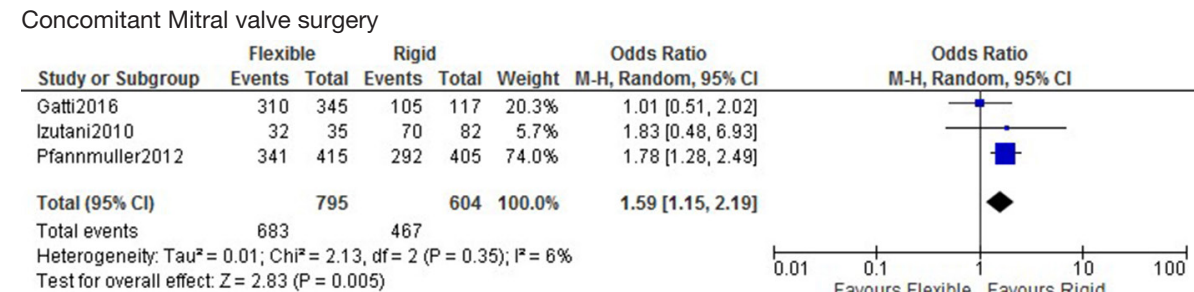

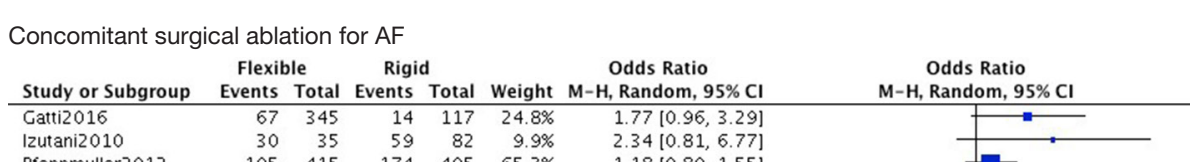

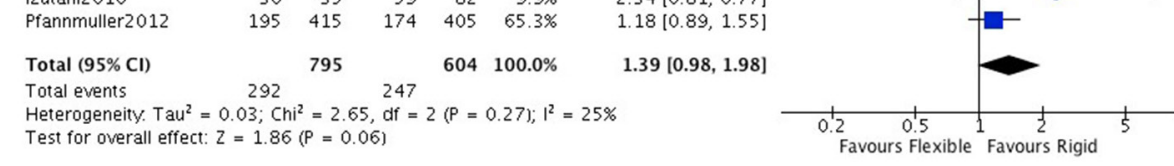

Figure S2 Comparison of perioperative variables in flexible band and rigid ring.

$\underset{1 \text { year }}{\text { A }}$

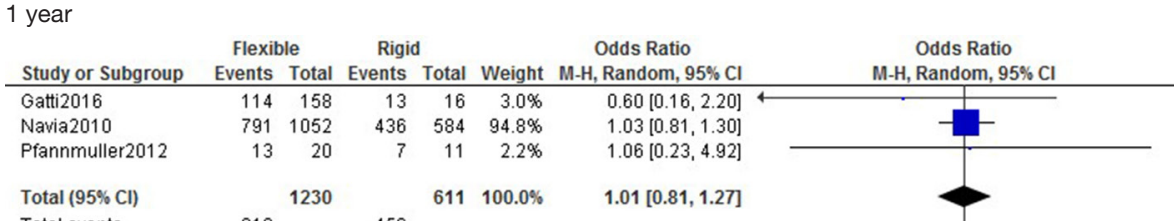

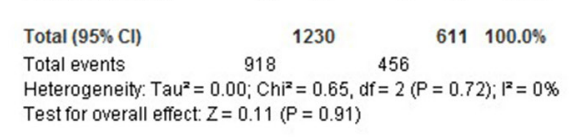

$\underset{1 \text { year }}{\mathrm{B}}$

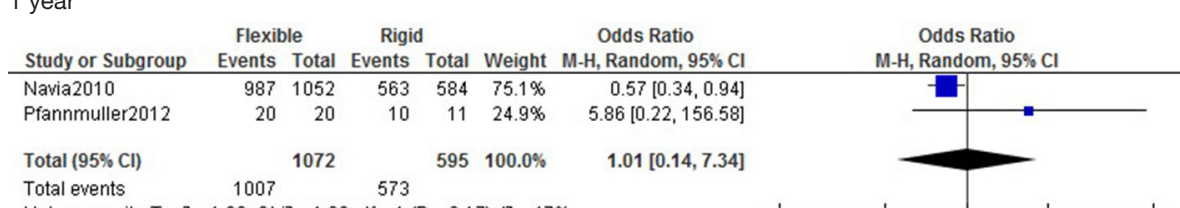

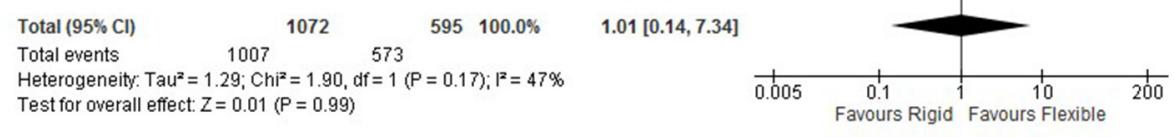

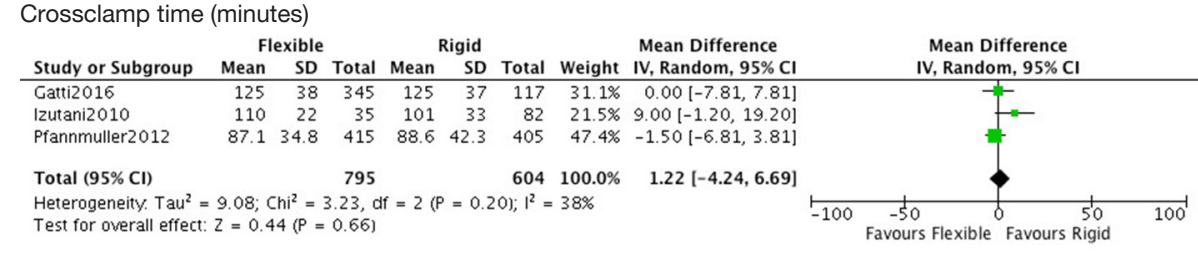

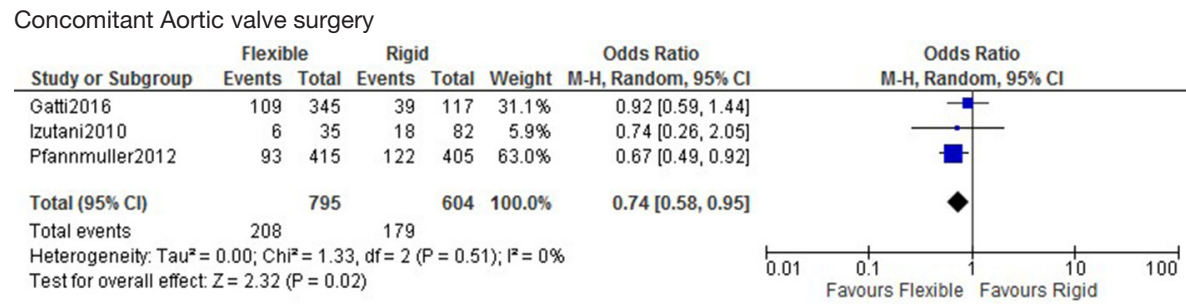

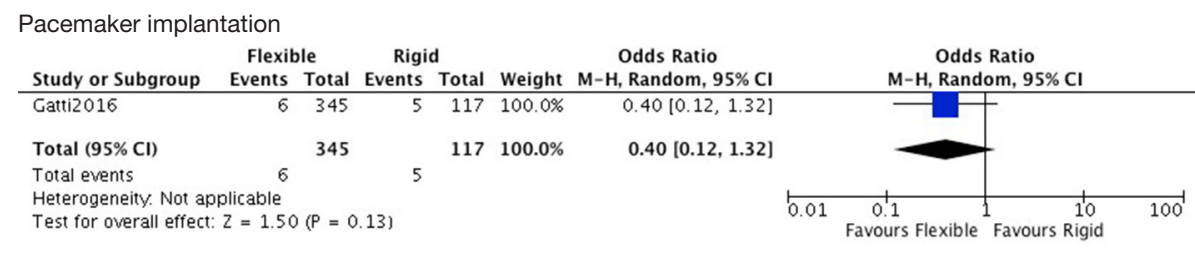

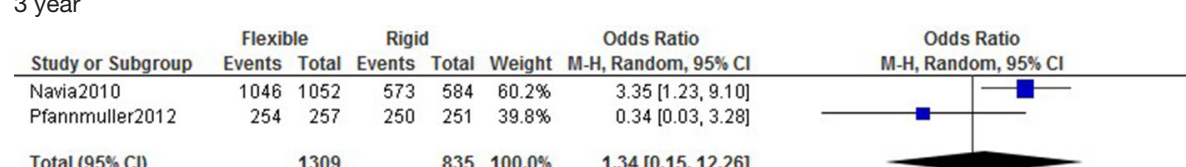

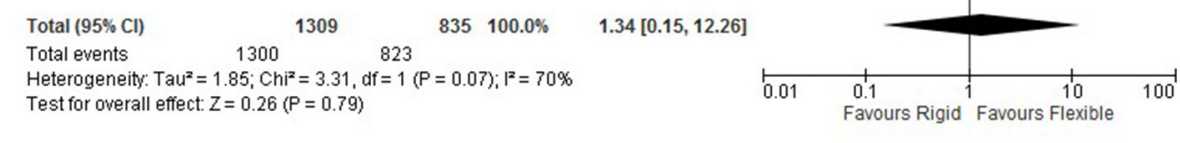

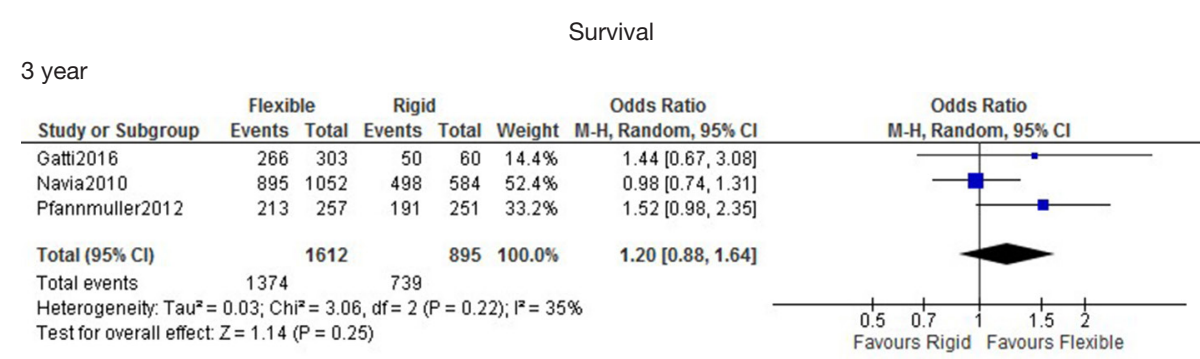

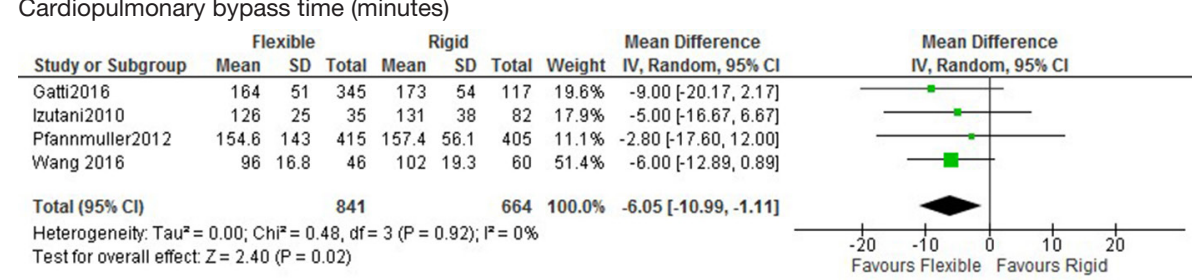

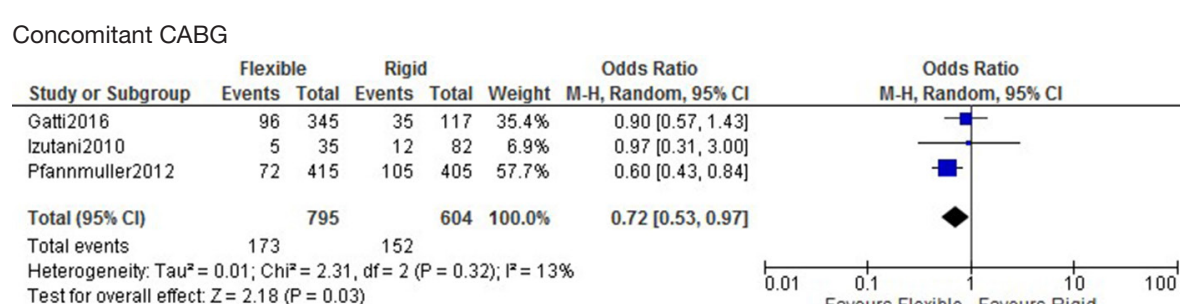

5 year

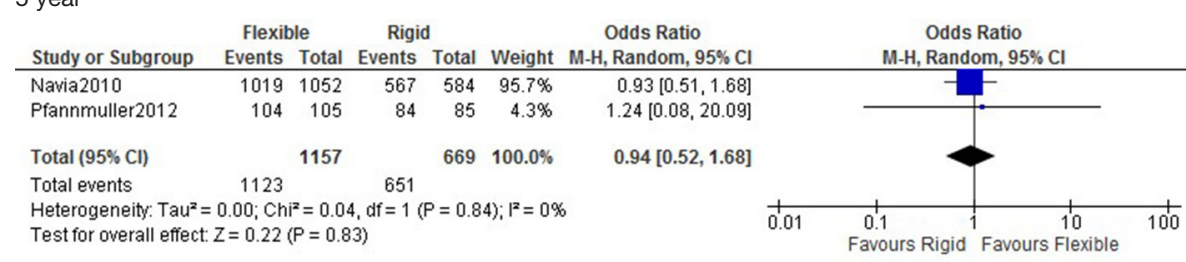
5 year

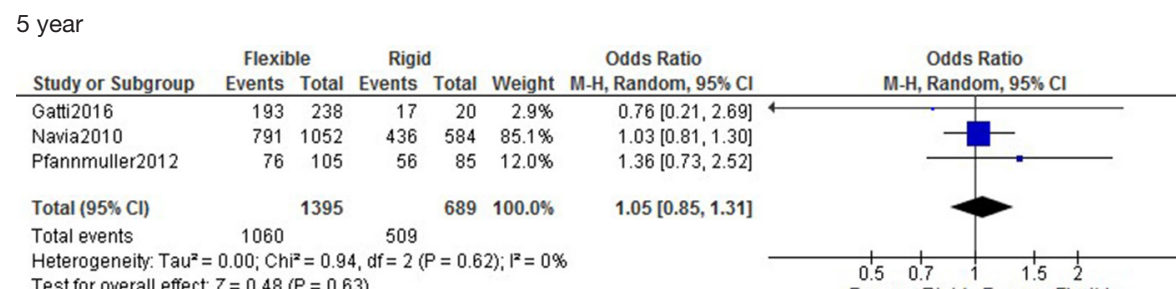

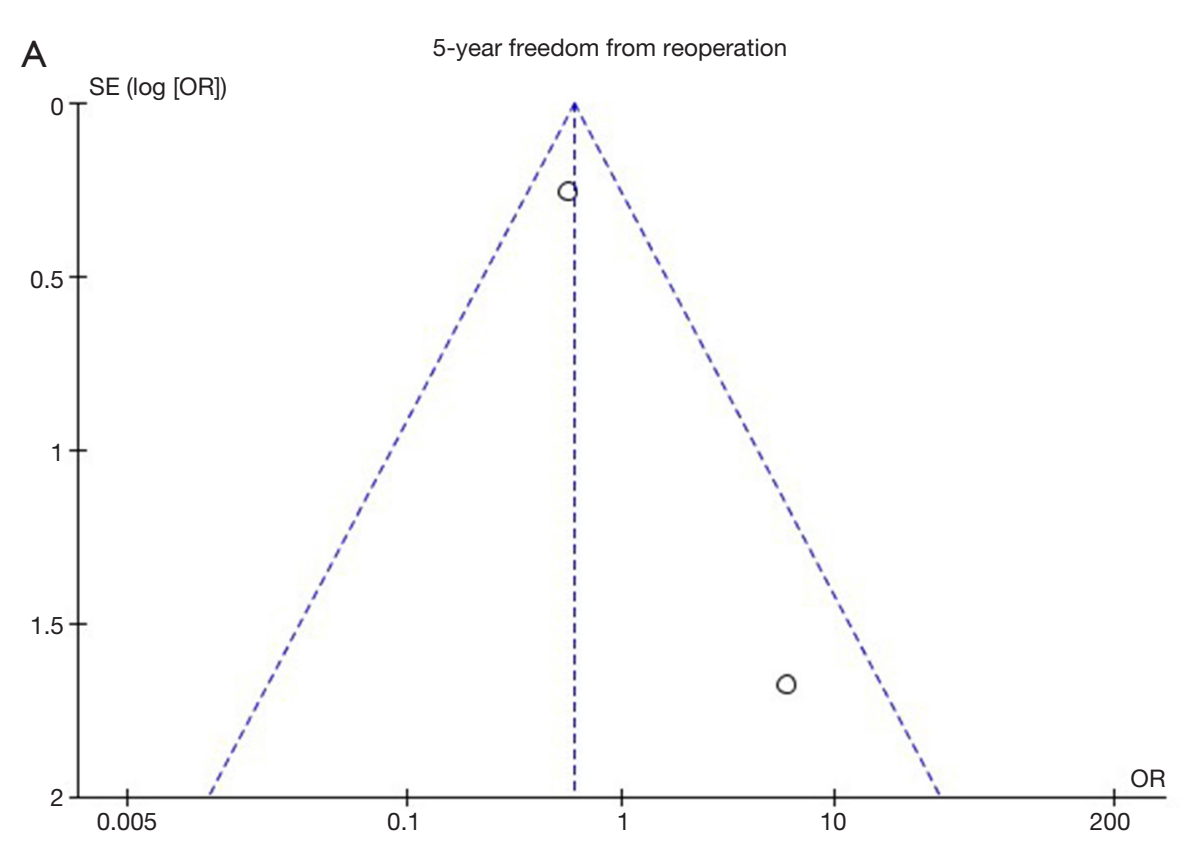
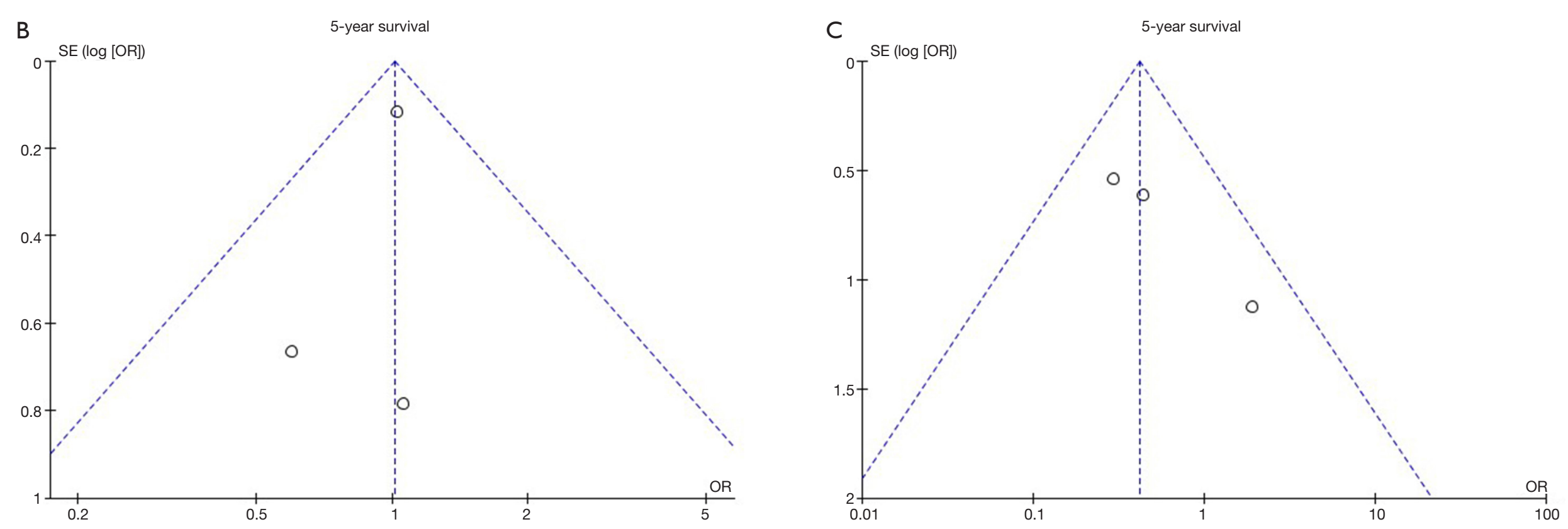

Figure 54 Publication bias assessment via funnel plots. 